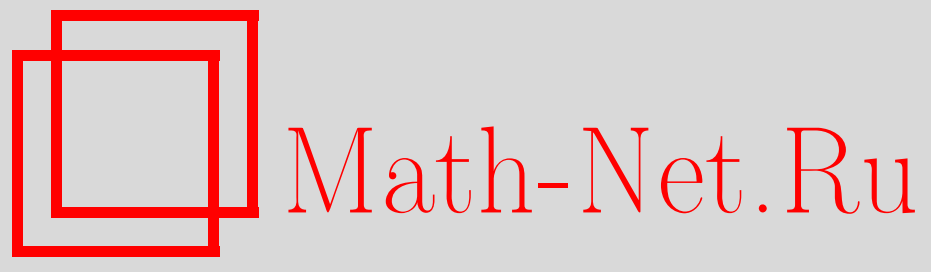

В. А. Андреев, Обобщенное неравенство Белла и метод его проверки, ТМФ, 2007, том 152, номер 3, 488-501

DOI: https://doi.org/10.4213/tmf6105

Использование Общероссийского математического портала Math-Net.Ru подразумевает, что вы прочитали и согласны с пользовательским соглашением http://www . mathnet.ru/rus/agreement

Параметры загрузки:

IP: 18.234 .197 .8

26 апреля 2023 г., 12:41:04

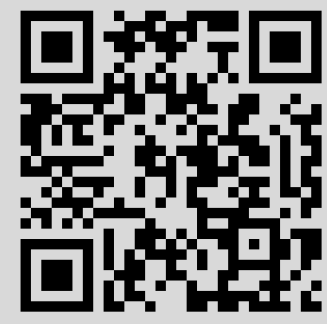




\title{
ОБОБЩЕННОЕ НЕРАВЕНСТВО БЕЛЛА И МЕТОД ЕГО ПРОВЕРКИ
}

\begin{abstract}
С помощью редукции матрицы плотности двучастичного спинового состояния построено обобщенное неравенство Белла-Клаузера-Хорне-Шимони-Хольта. Для каждого конкретного состояния при специальном выборе векторов $\vec{a}$, $\vec{b}, \vec{c}, \vec{d}$ оно превращается в точное равенство. Показано, как с помощью редуцированной матрицы плотности можно находить такие векторы. Обе части этого равенства имеют конкретное численное значение. Указано на связь этого числа с мерой запутанности двучастичного спинового состояния.
\end{abstract}

Ключевые слова: квантовая механика, запутанные состояния, матрица плотности, неравенство Белла.

\section{1. ВВЕДЕНИЕ}

Неравенства Белла-Клаузера-Хорне-Шимони-Хольта (БКХШХ) [1], [2] широко используются для анализа свойств запутанности двучастичных спиновых состояний. Их левая часть выражается через средние значения спиновой корреляции. Каждая такая величина $E(\vec{a}, \vec{b})$ зависит от двух единичных векторов, задающих направления в пространстве, для которых измеряют проекции спинов частиц, образующих коррелированную пару. Вся левая часть неравенства зависит от четырех единичных векторов $\vec{a}, \vec{b}, \vec{c}, \vec{d}$. Проверка неравенств БКХШХ состоит в том, что их левая часть (LH) измеряется при различных направлениях этих векторов и далее выбирается максимальное значение результата измерения. Если это максимальное значение LH > 2, то делается вывод, что для данного состояния неравенство БКХШХ нарушается. Такая проверка проводилась рядом экспериментальных групп [3]-[8]. При этом следует учитывать, что результат измерения зависит и от того, какое именно двучастичное состояние используется в эксперименте [9]. Факт нарушения неравенств БКХШХ обычно связывают с отсутствием в квантовой теории скрытых параметров, ее возможной нелокальностью и другими фундаментальными проблемами [10]-[18]. Кроме того, по результатам измерений можно получить ответ на вопрос о том, с какими состояниями имеют дело в эксперименте: запутанными, сепарабельными или факторизуемыми.

* Физический институт им. П. Н. Лебедева РАН, Москва, Россия. E-mail: andrvlad@yandex.ru 
Но, чтобы сделать определенный вывод, следует быть уверенным, что при измерениях был получен именно максимальный результат. Для этого желательно заранее знать, при каких положениях векторов $\vec{a}, \vec{b}, \vec{c}, \vec{d}$ этот результат достигается.

Неравенство БКХШХ, полученное в рамках модели скрытых переменных, имеет вид

$$
|E(\vec{a}, \vec{b})+E(\vec{a}, \vec{c})+E(\vec{d}, \vec{b})-E(\vec{d}, \vec{c})| \leqslant 2 .
$$

Оно выполняется для чистых факторизуемых состояний, и при определенных положениях векторов $\vec{a}, \vec{b}, \vec{c}, \vec{d}$ левая часть неравенства (1) достигает своего максимального значения - неравенство переходит в равенство. Неравенство (1) также выполняется для сепарабельных состояний и для некоторых классов запутанных смешанных состояний. При этом для них левая часть неравенства (1) может не только не достигать максимального значения, но и быть сколь угодно малой.

Более общее неравенство БКХШХ имеет вид

$$
|E(\vec{a}, \vec{b})+E(\vec{a}, \vec{c})+E(\vec{d}, \vec{b})-E(\vec{d}, \vec{c})| \leqslant 2 \sqrt{2} .
$$

Это неравенство выполняется для всех двучастичных спиновых состояний, однако не для каждого состояния его левая часть LH достигает максимального значения $2 \sqrt{2}$. Это значение можно получить только для чистых максимально запутанных состояний. Для всех остальных двучастичных спиновых состояний максимальное значение левой части неравенства (2) меньше $2 \sqrt{2}$. Интерпретация неравенства БКХШХ в рамках метода квантовой томографии обсуждалась в работе [19].

При экспериментальной проверке неравенства БКХШХ важно знать не только максимальное значение левой части неравенства $(2)$, но и те векторы $\vec{a}, \vec{b}, \vec{c}, \vec{d}$, при которых это значение достигается для данного состояния. Именно эти значения векторов и следует реализовать в эспериментальной установке.

В данной работе мы покажем, каким образом можно находить эти векторы для конкретных двучастичных спиновых состояний. Для этого будут использованы техника редукции матрицы плотности двучастичных спиновых состояний и построенное с ее помощью обобщенное неравенство БКХШХ. Это неравенство было предложено в работах [20], [21], но его строгого доказательства не приводилось. Здесь мы приводим это доказательство, которое одновременно дает и процедуру выбора оптимального набора векторов $\vec{a}, \vec{b}, \vec{c}, \vec{d}$.

Рассмотрены конкретные примеры двучастичных спиновых состояний, как чистых, так и смешанных. Указано также на связь величины LH с мерой запутанности двучастичного спинового состояния.

\section{2. ОБОБЩЕННЫЕ НЕРАВЕНСТВА БКХШХ}

Неравенства Белла строятся для наблюдаемой величины $E(\vec{a}, \vec{b})$, называемой спиновой корреляцией. Она определяется как учетверенное среднее значение произведений проекций спина первой частицы на ось $\vec{a}$, а второй частицы на ось $\vec{b}$.

Спиновая корреляция измеряется следующим образом. Пусть имеется источник пар коррелированных частиц со спином $S=1 / 2$ и две оси в конфигурационном 
пространстве, которые задаются единичными векторами $\vec{a}$ и $\vec{b}$. Последовательно проводится измерение проекций на эти оси спинов частиц из $N$ таких пар. После чего строится величина

$$
E_{N}(\vec{a}, \vec{b})=\frac{1}{N} \sum_{i=1}^{N} a_{i} b_{i} .
$$

Здесь $a_{i}=1$, если проекция спина первой частицы из $i$-й коррелированной пары на ось $\vec{a}$ равна $+1 / 2$, и $a_{i}=-1$, если проекция спина первой частицы из $i$-й коррелированной пары на ось $\vec{a}$ равна $-1 / 2$. Соответственно $b_{i}=1$, если проекция спина второй частицы из $i$-й коррелированной пары на ось $\vec{b}$ равна $+1 / 2$, и $b_{i}=-1$, если проекция спина второй частицы из $i$-й коррелированной пары на ось $\vec{b}$ равна $-1 / 2$.

Наблюдаемая величина $E_{N}$ называется спиновой корреляцией. Ее оператор для достаточно больших $N$ определяется следующим образом. Пусть имеется одно спиновое состояние такое, что направление спина вверх по оси $Z$ обозначается как $|+\rangle$, а вниз по оси $Z-$ как $|-\rangle$. На этих векторах спиновые операторы задаются соотношениями

$$
\begin{aligned}
& S_{x}|+\rangle=\frac{1}{2}|-\rangle, \quad S_{y}|+\rangle=\frac{i}{2}|-\rangle, \quad S_{z}|+\rangle=\frac{1}{2}|+\rangle, \\
& S_{x}|-\rangle=\frac{1}{2}|+\rangle, \quad S_{y}|-\rangle=-\frac{i}{2}|+\rangle, \quad S_{z}|-\rangle=-\frac{1}{2}|-\rangle .
\end{aligned}
$$

Оператор $\hat{a}$ удвоенной проекции спина на направление, задаваемое вектором $\vec{a}=$ $\left(a_{x}, a_{y}, a_{z}\right),|\vec{a}|=1$, имеет вид

$$
\hat{a}=2(\vec{a}, \vec{S})=2\left(a_{x} S_{x}+a_{y} S_{y}+a_{z} S_{z}\right) .
$$

Для двучастичных состояний можно определить оператор

$$
\hat{a} \otimes \hat{b}=4\left(\vec{a}, \vec{S}^{(1)}\right) \otimes\left(\vec{b}, \vec{S}^{(2)}\right),
$$

действующий в четырехмерном линейном пространстве, натянутом на базисные векторы

$$
\begin{array}{ll}
\Psi_{++}=|(1)+\rangle|(2)+\rangle, & \Psi_{+-}=|(1)+\rangle|(2)-\rangle, \\
\Psi_{-+}=|(1)-\rangle|(2)+\rangle, & \Psi_{--}=|(1)+\rangle|(2)+\rangle .
\end{array}
$$

При этом операторы $\vec{S}^{(1)}$ действуют на векторы $|(1)+\rangle,|(1)-\rangle$, а операторы $\vec{S}^{(2)}-$ на векторы $|(2)+\rangle,|(2)-\rangle$. Матричные элементы $\langle\psi|\hat{a} \otimes \hat{b}| \psi\rangle$ оператора (6) являются учетверенными средними значениями произведений проекций спина первой частицы на ось $\vec{a}$, а второй частицы на ось $\vec{b}$.

Оператор (6) соответствует наблюдаемой величине, называемой спиновой корреляцией. В базисе (7) оператор (6) имеет вид матрицы

$\hat{a} \otimes \hat{b}=$

$$
=\left(\begin{array}{cccc}
a_{z} b_{z} & a_{z}\left(b_{x}-i b_{y}\right) & \left(a_{x}-i a_{y}\right) b_{z} & \left(a_{x}-i a_{y}\right)\left(b_{x}-i b_{y}\right) \\
a_{z}\left(b_{x}+i b_{y}\right) & -a_{z} b_{z} & \left(a_{x}-i a_{y}\right)\left(b_{x}+i b_{y}\right) & -\left(a_{x}-i a_{y}\right) b_{z} \\
\left(a_{x}+i a_{y}\right) b_{z} & \left(a_{x}+i a_{y}\right)\left(b_{x}-i b_{y}\right) & -a_{z} b_{z} & -a_{z}\left(b_{x}-i b_{y}\right) \\
\left(a_{x}+i a_{y}\right)\left(b_{x}+i b_{y}\right) & -\left(a_{x}+i a_{y}\right) b_{z} & -a_{z}\left(b_{x}+i b_{y}\right) & a_{z} b_{z}
\end{array}\right) .
$$


Если спиновое двучастичное состояние в базисе (8) описывается матрицей плотности

$$
\rho=\left\|\rho_{i j}\right\|, \quad i, j=1,2,3,4,
$$

то значение величины спиновой корреляции имеет вид

$$
E(\vec{a}, \vec{b})=\operatorname{Sp}(\hat{a} \otimes \hat{b} \rho) .
$$

Для вычисления этой величины часто оказывается удобным использовать вместо матрицы плотности $\rho$ двучастичного спинового состояния ее редукцию $P$. Такая редукция есть $(3 \times 3)$-матрица

$$
\begin{aligned}
P & =\left\|P_{i j}\right\|= \\
& =\left(\begin{array}{ccc}
\rho_{14}+\rho_{23}+\rho_{32}+\rho_{41} & i\left(\rho_{14}-\rho_{23}+\rho_{32}-\rho_{41}\right) & \rho_{13}+\rho_{31}-\rho_{24}-\rho_{42} \\
i\left(\rho_{14}+\rho_{23}-\rho_{32}-\rho_{41}\right) & -\rho_{14}+\rho_{23}+\rho_{32}-\rho_{41} & i\left(\rho_{13}-\rho_{31}-\rho_{24}+\rho_{42}\right) \\
\rho_{12}+\rho_{21}-\rho_{34}-\rho_{43} & i\left(\rho_{12}-\rho_{21}-\rho_{34}+\rho_{43}\right) & \rho_{11}-\rho_{22}-\rho_{33}+\rho_{44}
\end{array}\right) .
\end{aligned}
$$

Используя редуцированную матрицу $P$, величину (10) можно представить в виде скалярного произведения векторов в трехмерном пространстве,

$$
E(\vec{a}, \vec{b})=\left(a_{x}, a_{y}, a_{z}\right) P\left(\begin{array}{l}
b_{x} \\
b_{y} \\
b_{z}
\end{array}\right)=(\vec{a}, P \vec{b}) .
$$

Редуцированная матрица $P$ не эквивалентна исходной матрице плотности $\rho$ : из формулы (11) легко видеть, что одной и той же матрице $P$ отвечают разные матрицы $\rho$, т.е. матрица $P$ не задает полностью двучастичное состояние. Однако той информации, которая в ней содержится, достаточно для того, чтобы вычислить величину спиновой корреляции $E(\vec{a}, \vec{b})$, соответствующую этому состоянию. Преимущество редуцированной матрицы $P$ состоит в том, что она, как правило, имеет более простой вид, чем исходная матрица плотности $\rho$, и пользоваться формулой (12) оказывается проще и удобнее, чем формулой (10). Ниже мы убедимся в этом на конкретных примерах.

ПРЕДЛОЖЕНИЕ. Для двучастичного спинового состояния, описъваемого матрищей плотности (9), справедливо обобщенное неравенство БКХШХ

$$
|E(\vec{a}, \vec{b})+E(\vec{a}, \vec{c})+E(\vec{d}, \vec{b})-E(\vec{d}, \vec{c})| \leqslant \sqrt{2} \sup _{n_{1}, n_{2}}\left(\left|P \vec{n}_{1}\right|+\left|P \vec{n}_{2}\right|\right) .
$$

Здесь точная верхняя грань берется по всем парам векторов $\vec{n}_{1}, \vec{n}_{2}$ таким, что

$$
\left(\vec{n}_{1}, \vec{n}_{2}\right)=0, \quad\left|\vec{n}_{1}\right|=\left|\vec{n}_{2}\right|=1 .
$$

ДокАзАТЕЛьСтво. Используя представление (12) для величины спиновой корреляции $E(\vec{a}, \vec{b})$, левую часть неравенства (13) представим в виде

$$
\begin{aligned}
& |E(\vec{a}, \vec{b})+E(\vec{a}, \vec{c})+E(\vec{d}, \vec{b})-E(\vec{d}, \vec{c})|= \\
& \quad=|(\vec{a}, P \vec{b})+(\vec{a}, P \vec{c})+(\vec{d}, P \vec{b})-(\vec{d}, P \vec{c})|=|(\vec{a}, P(\vec{b}+\vec{c}))+(\vec{d}, P(\vec{b}-\vec{c}))| .
\end{aligned}
$$


Векторы $\vec{b}+\vec{c}, \vec{b}-\vec{c}$ ортогональны друг другу, и сумма квадратов их модулей равна 4 :

$$
(\vec{b}+\vec{c}, \vec{b}-\vec{c})=0, \quad|(\vec{b}+\vec{c})|^{2}+|(\vec{b}-\vec{c})|^{2}=4 .
$$

При фиксированных векторах $\vec{b}, \vec{c}$ выражение (14) принимает наибольшее значение, когда вектор $\vec{a}$ параллелен вектору $P(\vec{b}+\vec{c})$, а вектор $\vec{d}$ параллелен вектору $P(\vec{b}-\vec{c})$. Пусть среди всех пар векторов $\vec{b}, \vec{c}$ мы выбрали такую, для которой величина (14) принимает наибольшее значение. Докажем лемму.

ЛЕмма. Векторы $\vec{b}+\vec{c}, \vec{b}-\vec{c}$ можно выбрать так, что их модули будут одинаковы, m.e.

$$
|(\vec{b}+\vec{c})|^{2}=|(\vec{b}-\vec{c})|^{2}=2 .
$$

ДоказАтЕЛЬСтво. Пусть векторы $\vec{b}+\vec{c}, \vec{b}-\vec{c}$ удовлетворяют условиям (15). Рассмотрим плоскость, в которой лежат эти векторы, и выберем базис в трехмерном пространстве так, чтобы векторы $\vec{k}_{1}, \vec{k}_{2}$ лежали в этой плоскости, а третий базисный вектор $\vec{k}_{3}$ был ортогонален векторам $\vec{k}_{1}, \vec{k}_{2}$. Пусть базисные векторы имеют вид

$$
\vec{k}_{1}=\left(\begin{array}{c}
\sin \varphi \\
\cos \varphi \\
0
\end{array}\right), \quad \vec{k}_{2}=\left(\begin{array}{c}
-\cos \varphi \\
\sin \varphi \\
0
\end{array}\right), \quad \vec{k}_{3}=\left(\begin{array}{l}
0 \\
0 \\
1
\end{array}\right)
$$

В базисе из этих векторов редуцированная матрица (11) записывается как

$$
P_{k}=\left\|k_{i j}\right\|=\left(\begin{array}{lll}
k_{11} & k_{12} & k_{13} \\
k_{21} & k_{22} & k_{23} \\
k_{31} & k_{32} & k_{33}
\end{array}\right) .
$$

Рассмотрим величину

$$
\mathrm{RH}=2\left(\left|P\left(\vec{k}_{1}\right)\right| \cos \psi+\left|P\left(\vec{k}_{2}\right)\right| \sin \psi\right)=2 G \sin (\phi+\psi) .
$$

Здесь

$$
\begin{aligned}
\sin \phi=\frac{\left|P\left(k_{1}\right)\right|}{G}, \quad \cos \phi=\frac{\left|P\left(k_{2}\right)\right|}{G}, \quad G=\sqrt{k_{11}^{2}+k_{21}^{2}+k_{31}^{2}+k_{12}^{2}+k_{22}^{2}+k_{32}^{2}}, \\
\left|P\left(k_{1}\right)\right|=\left[\left(k_{11}^{2}+k_{21}^{2}+k_{31}^{2}\right) \sin ^{2} \varphi+\left(k_{12}^{2}+k_{22}^{2}+k_{32}^{2}\right) \cos ^{2} \varphi+\right. \\
\left.\quad+\left(k_{11} k_{12}+k_{21} k_{22}+k_{31} k_{32}\right) \sin 2 \varphi\right]^{1 / 2}, \\
\left|P\left(k_{2}\right)\right|=\left[\left(k_{11}^{2}+k_{21}^{2}+k_{31}^{2}\right) \cos ^{2} \varphi+\left(k_{12}^{2}+k_{22}^{2}+k_{32}^{2}\right) \sin ^{2} \varphi-\right. \\
\\
\left.\quad-\left(k_{11} k_{12}+k_{21} k_{22}+k_{31} k_{32}\right) \sin 2 \varphi\right]^{1 / 2} .
\end{aligned}
$$

Выражение (19) - это правая часть неравенства (13), вычисленная для двух векторов, удовлетворяющих условию (15). Она достигает максимального значения, когда $\phi+\psi=\pi / 2$. Таким образом, имеется целое семейство пар векторов $\sqrt{2} \vec{k}_{1} \cos \psi$, $\sqrt{2} \vec{k}_{2} \sin \psi$, на которых достигается максимум выражения (19). Их модули, вообще говоря, различны, но в этом семействе существует пара векторов с равными модулями. Найдем ее явный вид. 
Для равенства модулей векторов пары должно выполняться равенство $\psi=\pi / 4$. Тогда из условия максимальности RН следует, что и $\phi=\pi / 4$. Угол $\phi$ задается соотношениями (20), из которых следует, что в этом случае должно выполняться условие

$$
\left|P\left(\vec{k}_{1}\right)\right|^{2}=\left|P\left(\vec{k}_{2}\right)\right|^{2} .
$$

Это равенство выполняется при определенных значениях угла $\varphi$, которые можно найти, используя выражения (20):

$$
\begin{gathered}
\left(k_{11}^{2}+k_{21}^{2}+k_{31}^{2}-k_{12}^{2}-k_{22}^{2}-k_{32}^{2}\right) \sin ^{2} \varphi-\left(k_{11}^{2}+k_{21}^{2}+k_{31}^{2}-k_{12}^{2}+k_{22}^{2}+k_{32}^{2}\right) \cos ^{2} \varphi= \\
=2 \sin 2 \varphi\left(k_{11} k_{12}+k_{21} k_{22}^{2}+k_{31} k_{32}\right) .
\end{gathered}
$$

Отсюда получаем, что

$$
\operatorname{tg} 2 \varphi=\frac{k_{12}^{2}+k_{22}^{2}+k_{32}^{2}-k_{11}^{2}-k_{21}^{2}-k_{31}^{2}}{2\left(k_{11} k_{12}+k_{21} k_{22}^{2}+k_{31} k_{32}\right)} .
$$

Это равенство задает то значение угла $\varphi$, при котором единичные векторы $k_{1}$ и $k_{2}$ в (17) образуют пару, на которой величина (19) достигает максимального значения. Мы показали, что в качестве векторов $\vec{b}+\vec{c}, \vec{b}-\vec{c}$ можно взять векторы $\sqrt{2} \vec{k}_{1}, \sqrt{2} \vec{k}_{2}$ у которых значение угла $\varphi$ определяется выражением (22). Таким образом, лемма доказана.

Условие (22) задает то значение угла $\varphi$, при котором единичные векторы (17) $\vec{k}_{1}, \vec{k}_{2}$ образуют пару, на которой величина (19) достигает максимального значения. Поэтому теперь для того, чтобы получить утверждение, содержащееся в предложении, достаточно заметить, что правая часть неравенства (13) принимает свое максимальное значение, когда $\vec{n}_{1}=\vec{k}_{1}, \vec{n}_{2}=\vec{k}_{2}$. Это максимальное значение равно $2 G$. Обобщенное неравенство БКХШХ принимает вид

$$
|E(\vec{a}, \vec{b})+E(\vec{a}, \vec{c})+E(\vec{d}, \vec{b})-E(\vec{d}, \vec{c})| \leqslant 2 G=2 \sqrt{k_{11}^{2}+k_{21}^{2}+k_{31}^{2}+k_{12}^{2}+k_{22}^{2}+k_{32}^{2}} .
$$

Здесь $k_{i j}$ - элементы редуцированной матрицы (11), записанной в базисе (17).

Итак, мы доказали обобщенное неравенство БКХШХ (13). У этого неравенства и левая, и правая части зависят от матрицы плотности двучастичного спинового состояния. Поэтому в отличие от неравенства (2) при подстановке в обобщенное неравенство БКХШХ векторов $\vec{a}, \vec{b}, \vec{c}, \vec{d}$, реализующих максимум правой части, оно превращается в равенство.

Перейдем теперь непосредственно к описанию процедуры вычислений.

\section{3. ВЫЧИСЛЕНИЕ ОПТИМАЛЬНОГО ПОЛОЖЕНИЯ ВЕКТОРОВ $\vec{a}, \vec{b}, \vec{c}, \vec{d}$}

Процедура доказательства неравенства (13) фактически дает нам также способ, позволяющий найти нужный набор векторов $\vec{a}, \vec{b}, \vec{c}, \vec{d}$. Алгоритм поиска состоит из следующих операций. 
1. По матрице плотности двучастичного спинового состояния $\rho$ строим редуцированную матрицу $P(11)$.

2. Ищем два ортогональных единичных вектора $\vec{n}_{1}, \vec{n}_{2}$ таких, что величина $\operatorname{RH}\left(\vec{n}_{1}, \vec{n}_{2}\right)=\sqrt{2}\left(\left|P \vec{n}_{1}\right|+\left|P \vec{n}_{2}\right|\right)$ принимает наибольшее значение. Это значение мы обозначаем RH.

3. По этим векторам $\vec{n}_{1}, \vec{n}_{2}$ строим векторы $\vec{b}, \vec{c}$ :

$$
\vec{b}=\frac{\vec{n}_{1}+\vec{n}_{2}}{\sqrt{2}}, \quad \vec{c}=\frac{\vec{n}_{1}-\vec{n}_{2}}{\sqrt{2}} .
$$

4. Действуем на векторы $\vec{n}_{1}, \vec{n}_{2}$ матрицей (11), получаем векторы $P \vec{n}_{1}, P \vec{n}_{2}$ и строим по ним векторы

$$
\vec{a}=\frac{P \vec{n}_{1}}{\left|P \vec{n}_{1}\right|}, \quad \vec{d}=\frac{P \vec{n}_{2}}{\left|P \vec{n}_{2}\right|} .
$$

Рассмотрим некоторые частные случаи.

СлучАй 1. Пусть матрица $P$ имеет диагональную форму,

$$
P=\left(\begin{array}{ccc}
\lambda_{1} & 0 & 0 \\
0 & \lambda_{2} & 0 \\
0 & 0 & \lambda_{3}
\end{array}\right), \quad \lambda_{1} \geqslant \lambda_{2} \geqslant \lambda_{3}
$$

тогда ее действие на единичный вектор $\vec{n}_{1}$ запишется как

$$
P \vec{n}_{1}=\left(\begin{array}{ccc}
\lambda_{1} & 0 & 0 \\
0 & \lambda_{2} & 0 \\
0 & 0 & \lambda_{3}
\end{array}\right)\left(\begin{array}{c}
\cos \varphi \sin \theta \\
\sin \varphi \sin \theta \\
\cos \theta
\end{array}\right)=\left(\begin{array}{c}
\lambda_{1} \cos \varphi \sin \theta \\
\lambda_{2} \sin \varphi \sin \theta \\
\lambda_{3} \cos \theta
\end{array}\right) .
$$

Квадрат модуля вектора $P \vec{n}_{1}$ равен

$$
\begin{aligned}
& \lambda_{1}^{2} \cos ^{2} \varphi \sin ^{2} \theta+\lambda_{2}^{2} \sin ^{2} \varphi \sin ^{2} \theta+\lambda_{3}^{2} \cos ^{2} \theta= \\
& \quad=\lambda_{1}^{2}-\left(\lambda_{1}^{2}-\lambda_{2}^{2}\right) \sin ^{2} \varphi \sin ^{2} \theta-\left(\lambda_{1}^{2}-\lambda_{3}^{2}\right) \cos ^{2} \theta .
\end{aligned}
$$

Отсюда видно, что максимальное значение модуля достигается при $\cos ^{2} \theta=0$. Поэтому при нахождении векторов, на которых достигается максимальное значение левой части обобщенного неравенства БКХШХ (13), достаточно ограничиться теми векторами, у которых $\cos ^{2} \theta=0$. Рассмотрим два таких вектора, ортогональных друг другу:

$$
\vec{n}_{1}=\left(\begin{array}{c}
\cos \varphi \\
\sin \varphi \\
0
\end{array}\right), \quad \vec{n}_{2}=\left(\begin{array}{c}
\sin \varphi \\
-\cos \varphi \\
0
\end{array}\right)
$$

Мы будем искать векторы $\vec{n}_{1}, \vec{n}_{2}$, для которых величина $\operatorname{RH}\left(\vec{n}_{1}, \vec{n}_{2}\right)$ будет принимать наибольшее значение,

$$
\operatorname{RH}\left(\vec{n}_{1}, \vec{n}_{2}\right)=\sqrt{2}\left(\sqrt{\lambda_{1}^{2} \cos ^{2} \varphi+\lambda_{2}^{2} \sin ^{2} \varphi}+\sqrt{\lambda_{1}^{2} \sin ^{2} \varphi+\lambda_{2}^{2} \cos ^{2} \varphi}\right) .
$$

Величина $\operatorname{RH}\left(\vec{n}_{1}, \vec{n}_{2}\right)$ принимает свое максимальное значение при $\varphi=\pi / 4$. В этом случае

$$
\mathrm{RH}=2 \sqrt{\lambda_{1}^{2}+\lambda_{2}^{2}}
$$


Это значение достигается на единичных векторах

$$
\vec{n}_{1}=\frac{1}{\sqrt{2}}\left(\begin{array}{l}
1 \\
1 \\
0
\end{array}\right), \quad \vec{n}_{2}=\frac{1}{\sqrt{2}}\left(\begin{array}{c}
1 \\
-1 \\
0
\end{array}\right) .
$$

Отметим, что векторы $\vec{n}_{1}, \vec{n}_{2}$ имеют данный вид в том базисе, в котором редуцированная матрица $P(11)$ диагональна, см. (26).

По этим векторам $\vec{n}_{1}, \vec{n}_{2}$ с помощью формул (24), (25) легко определить векторы $\vec{a}, \vec{b}, \vec{c}, \vec{d}$.

СлучАй 2. Пусть редуцированную матрицу $P$ можно привести к нормальной форме, содержащей две жордановы клетки:

$$
P=\left(\begin{array}{ccc}
\lambda_{1} & 0 & 0 \\
0 & \lambda_{2} & 1 \\
0 & 0 & \lambda_{2}
\end{array}\right), \quad \lambda_{1} \geqslant \lambda_{2}
$$

Эта матрица имеет собственный вектор $\vec{k}_{1}=\left(\begin{array}{l}1 \\ 0 \\ 0\end{array}\right)$. Ограничим матрицу (32) на подпространство, ортогональное вектору $\vec{k}_{1}$ и рассмотрим вектор

$$
\vec{l}=p \vec{v}_{1}=\left(\begin{array}{cc}
\lambda_{2} & 1 \\
0 & \lambda_{2}
\end{array}\right)\left(\begin{array}{c}
\sin \varphi \\
\cos \varphi
\end{array}\right)=\left(\begin{array}{c}
\lambda_{2} \sin \varphi+\cos \varphi \\
\lambda_{2} \cos \varphi
\end{array}\right) .
$$

Найдем теперь такой угол $\varphi$, при котором вектор $\vec{l}$ имеет наибольший модуль,

$$
\begin{aligned}
|\vec{l}|^{2} & =\lambda_{2}^{2} \sin ^{2} \varphi+2 \lambda_{2} \sin \varphi \cos \varphi+\cos ^{2} \varphi+\lambda_{2}^{2} \cos ^{2} \varphi=\lambda_{2}^{2}+\lambda_{2} \sin 2 \varphi+\frac{1}{2}+\frac{1}{2} \cos 2 \varphi= \\
& =\lambda_{2}^{2}+\frac{1}{2}+\sqrt{\lambda_{2}^{2}+\frac{1}{4}} \sin (\phi+2 \varphi),
\end{aligned}
$$

где

$$
\sin \phi=\frac{1}{2 \sqrt{\lambda_{2}^{2}+1 / 4}}, \quad \cos \phi=\frac{\lambda_{2}}{\sqrt{\lambda_{2}^{2}+1 / 4}} .
$$

Мы видим, что модуль вектора (33) принимает наибольшее значение

$$
\left|\vec{l}_{\mathrm{M}}\right|^{2}=\lambda_{2}^{2}+\frac{1}{2}+\sqrt{\lambda_{2}^{2}+\frac{1}{4}},
$$

если

$$
\varphi=\frac{\pi}{4}-\frac{\phi}{2}
$$

Рассмотрим теперь два ортогональных вектора

$$
\vec{v}_{1}=\left(\begin{array}{c}
\sin \varphi \\
\cos \varphi
\end{array}\right), \quad \vec{v}_{2}=\left(\begin{array}{c}
\cos \varphi \\
-\sin \varphi
\end{array}\right) .
$$


В базисе, образованном этими векторами $\vec{v}_{1}, \vec{v}_{2}$ рассмотрим пару ортогональных векторов, сумма квадратов модулей которых равна 2. Эту пару выберем в виде $\left(\vec{v}_{1} \cos \gamma, \vec{v}_{2} \sin \gamma\right)$, где $\gamma-$ произвольный угол. Определим, какое максимальное значение принимает величина

$$
\begin{aligned}
\mathrm{RH} & =2\left|p \vec{v}_{1}\right| \cos \gamma+2\left|p \vec{v}_{1}\right| \sin \gamma= \\
& =2 \sqrt{\lambda_{2}^{2}+2 \lambda_{2} \sin \varphi \cos \varphi+\cos ^{2} \varphi} \cdot \cos \gamma+\sqrt{\lambda_{2}^{2}-2 \lambda_{2} \sin \varphi \cos \varphi+\sin ^{2} \varphi} \cdot \sin \gamma= \\
& =2 \sqrt{2 \lambda_{2}^{2}+1} \cdot \sin (\gamma+\psi) .
\end{aligned}
$$

Здесь

$$
\begin{aligned}
\sin \psi & =\frac{\sqrt{\lambda_{2}^{2}+2 \lambda_{2} \sin \varphi \cos \varphi+\cos ^{2} \varphi}}{\sqrt{2 \lambda_{2}^{2}+1}}, \\
\cos \psi & =\frac{\sqrt{\lambda_{2}^{2}-2 \lambda_{2} \sin \varphi \cos \varphi+\sin ^{2} \varphi}}{\sqrt{2 \lambda_{2}^{2}+1}} .
\end{aligned}
$$

Из формулы (35) видно, что максимальное значение величины RH при условии, что для вычисления используются только векторы, ортогональные вектору $\vec{k}_{1}$, равно $\mathrm{RH}=2 \sqrt{2 \lambda_{2}^{2}+1}$. Оно достигается при

$$
\gamma+\psi=\frac{\pi}{2}
$$

При фиксированном угле $\varphi$ фиксирован и угол $\psi$; по заданным $\varphi, \psi$ с помощью условия (37) определяется угол $\phi$. Из формулы (37) следует, что если $\psi=\pi / 4$, то и $\gamma=\pi / 4$. Отсюда и из формул (36) получаем, что $\operatorname{tg} 2 \varphi=-1 /\left(2 \lambda_{2}\right)$.

Таким образом, среди множества пар ортогональных векторов, на которых достигается максимальное значение $\mathrm{RH}=2 \sqrt{2 \lambda_{2}^{2}+1}$, имеется и пара единичных векторов. Эти векторы имеют вид

$$
\vec{n}_{1}=\frac{1}{\sqrt{2}}\left(\begin{array}{c}
1 \\
\sin \varphi \\
\cos \varphi
\end{array}\right), \quad \vec{n}_{2}=\frac{1}{\sqrt{2}}\left(\begin{array}{c}
1 \\
-\cos \varphi \\
\sin \varphi
\end{array}\right)
$$

Здесь угол $\varphi$ определяется условием $\operatorname{tg} 2 \varphi=-1 /\left(2 \lambda_{2}\right)$.

Рассмотрим теперь пару ортогональных векторов вида

$$
\vec{s}_{1}=\vec{L}_{\mathrm{M}} \cos \delta+\vec{k}_{1} \sin \delta, \quad \vec{s}_{2}=\vec{L}_{\mathrm{M}} \sin \delta-\vec{k}_{1} \cos \delta
$$

где $\vec{L}_{\mathrm{M}}=\left(\begin{array}{c}0 \\ \vec{l}_{\mathrm{M}}\end{array}\right)$. Вектор $\vec{l}_{M}$ здесь тот же, что и в формуле (34). Из всех векторов вида (33) он обладает наибольшим модулем. Вычислим величину

$$
\begin{aligned}
\mathrm{RH} & =2\left|P \vec{s}_{1}\right| \cos \beta+2\left|P \vec{s}_{2}\right| \sin \beta= \\
& =\sqrt{\lambda_{1}^{2} \sin ^{2} \delta+\left|\vec{l}_{\mathrm{M}}\right|^{2} \cos ^{2} \delta} \cos \beta+\sqrt{\lambda_{1}^{2} \cos ^{2} \delta+\left|\vec{l}_{\mathrm{M}}\right|^{2} \sin ^{2} \delta} \sin \beta=
\end{aligned}
$$




$$
=\sqrt{\lambda_{1}^{2}+\left|\vec{l}_{\mathrm{M}}\right|^{2}} \sin (\beta+\psi)
$$

Здесь

$$
\sin \psi=\frac{\sqrt{\lambda_{1}^{2} \sin ^{2} \delta+\left|\vec{l}_{\mathrm{M}}\right|^{2} \cos ^{2} \delta}}{\sqrt{\lambda_{1}^{2}+\left|\vec{l}_{\mathrm{M}}\right|^{2}}}, \quad \cos \psi=\frac{\sqrt{\lambda_{1}^{2} \cos ^{2} \delta+\left|\vec{l}_{\mathrm{M}}\right|^{2} \sin ^{2} \delta}}{\sqrt{\lambda_{1}^{2}+\left|\vec{l}_{\mathrm{M}}\right|^{2}}},
$$

$\beta$ - произвольный угол. Величина RH (40) принимает свое максимальное значение при

$$
\beta+\psi=\frac{\pi}{2} .
$$

Из этой формулы следует, что если $\delta=\pi / 4$, то и $\psi=\pi / 4$, следовательно, $\beta=$ $\pi / 4$. Таким образом, среди множества пар ортогональных векторов, на которых достигается максимальное значение

$$
\mathrm{RH}=2 \sqrt{\lambda_{1}^{2}+\left|\vec{l}_{\mathrm{M}}\right|^{2}}=2 \sqrt{\lambda_{1}^{2}+\lambda_{2}^{2}+\frac{1}{2}+\sqrt{\lambda_{2}^{2}+\frac{1}{4}}},
$$

существует пара единичных векторов. Эти векторы имеют вид

$$
\vec{s}_{1}=\frac{1}{\sqrt{2}}\left(\begin{array}{c}
1 \\
\sin \varphi \\
\cos \varphi
\end{array}\right), \quad \vec{s}_{2}=\frac{1}{\sqrt{2}}\left(\begin{array}{c}
-1 \\
\sin \varphi \\
\cos \varphi
\end{array}\right)
$$

где угол $\varphi$ определен равенством (34).

Таким образом, мы имеем два максимальных значения величины RH. Одно из них достигается на векторах (32), а другое - на векторах (43). Каким из них следует пользоваться, зависит от величин $\lambda_{1}, \lambda_{2}$. Если $\left(\lambda_{1}^{2}-\lambda_{2}^{2}\right)^{2} \geqslant \lambda_{1}^{2}$, то $\sqrt{2 \lambda_{2}^{2}+1} \geqslant$ $\sqrt{\lambda_{1}^{2}+\lambda_{2}^{2}+1 / 2+\sqrt{\lambda_{2}^{2}+1 / 4}}$, и следует пользоваться парой векторов (37). Если $\left(\lambda_{1}^{2}-\lambda_{2}^{2}\right)^{2} \leqslant \lambda_{1}^{2}$, то $\sqrt{2 \lambda_{2}^{2}+1} \leqslant \sqrt{\lambda_{1}^{2}+\lambda_{2}^{2}+1 / 2+\sqrt{\lambda_{2}^{2}+1 / 4}}$, и следует пользоваться парой векторов (43).

\section{4. ПРИМЕРЫ}

Рассмотрим скалярное двучастичное состояние, которое также часто называют ЭПР-состоянием

ПримеР 1. Рассмотрим $\Psi_{0,0}=\frac{1}{\sqrt{2}}(|+\rangle|-\rangle-|-\rangle|+\rangle)$. Для этого состояния

$$
\rho_{0,0}=\frac{1}{2}\left(\begin{array}{cccc}
0 & 0 & 0 & 0 \\
0 & 1 & -1 & 0 \\
0 & -1 & 1 & 0 \\
0 & 0 & 0 & 0
\end{array}\right), \quad P_{0,0}=\left(\begin{array}{ccc}
-1 & 0 & 0 \\
0 & -1 & 0 \\
0 & 0 & -1
\end{array}\right) \text {. }
$$

Матрица $P$ диагональна, и можно воспользоваться формулой (30). Поэтому для такого состояния максимальное значение правой части неравенства (13) равно

$$
\mathrm{RH}=2 \sqrt{2} .
$$


Это значение достигается на любой паре векторов $\vec{n}_{1}, \vec{n}_{2}$, ортогональных друг другу: $\left(\vec{n}_{1}, \vec{n}_{2}\right)=0$. Четыре единичных вектора $\vec{a}, \vec{b}, \vec{c}, \vec{d}$ можно выразить через векторы $\vec{n}_{1}, \vec{n}_{2}$ следующим образом:

$$
\vec{a}=\vec{n}_{1}, \quad \vec{b}=\frac{\vec{n}_{1}+\vec{n}_{2}}{\sqrt{2}}, \quad \vec{c}=\frac{\vec{n}_{1}-\vec{n}_{2}}{\sqrt{2}}, \quad \vec{d}=\vec{n}_{2} .
$$

ПримеР 2. Такой же результат получается для $\Psi_{1,0}=\frac{1}{\sqrt{2}}(|+\rangle|-\rangle+|-\rangle|+\rangle)$. Для этого состояния

$$
\rho_{1,0}=\frac{1}{2}\left(\begin{array}{cccc}
0 & 0 & 0 & 0 \\
0 & 1 & 1 & 0 \\
0 & 1 & 1 & 0 \\
0 & 0 & 0 & 0
\end{array}\right), \quad P_{1,0}=\left(\begin{array}{ccc}
1 & 0 & 0 \\
0 & 1 & 0 \\
0 & 0 & -1
\end{array}\right) .
$$

Для состояния $\Psi_{1,0}$ векторы $\vec{a}, \vec{b}, \vec{c}, \vec{d}$ могут быть выражены через векторы $\vec{n}_{1}, \vec{n}_{2}$ точно так же, как и в случае состояния $\Psi_{0,0}$,

$$
\mathrm{RH}=2 \sqrt{2}, \quad \vec{a}=\vec{n}_{1}, \quad \vec{b}=\frac{\vec{n}_{1}+\vec{n}_{2}}{\sqrt{2}}, \quad \vec{c}=\frac{\vec{n}_{1}-\vec{n}_{2}}{\sqrt{2}}, \quad \vec{d}=\vec{n}_{2} .
$$

Векторы $\vec{n}_{1}, \vec{n}_{2}$ произвольны лишь с тем ограничением, что $\left(\vec{n}_{1}, \vec{n}_{2}\right)=0$.

ПримеР 3. До сих пор мы рассматривали чистые состояния. Рассмотрим сейчас состояние Вернера [22]. Это смешанное состояние, которое описывается матрицей плотности

$$
\rho_{\mathrm{W}}=\frac{1}{8}\left(\begin{array}{cccc}
1 & 0 & 0 & 0 \\
0 & 3 & -2 & 0 \\
0 & -2 & 3 & 0 \\
0 & 0 & 0 & 1
\end{array}\right)
$$

Для этого состояния матрица $P$ имеет вид

$$
P_{\mathrm{W}}=\frac{1}{2}\left(\begin{array}{ccc}
-1 & 0 & 0 \\
0 & -1 & 0 \\
0 & 0 & -1
\end{array}\right)
$$

$\mathrm{C}$ помощью этой матрицы можно найти, что для состояния (45) $\mathrm{RH}=\sqrt{2}$, и это максимальное значение достигается на каждой паре ортогональных векторов $\vec{n}_{1}, \vec{n}_{2}$ таких, что $\left(\vec{n}_{1}, \vec{n}_{2}\right)=0$. Четыре единичных вектора $\vec{a}, \vec{b}, \vec{c}, \vec{d}$ могут быть выражены через векторы $\vec{n}_{1}, \vec{n}_{2}$ следующим образом:

$$
\vec{a}=\vec{n}_{1}, \quad \vec{b}=\frac{\vec{n}_{1}+\vec{n}_{2}}{\sqrt{2}}, \quad \vec{c}=\frac{\vec{n}_{1}-\vec{n}_{2}}{\sqrt{2}}, \quad \vec{d}=\vec{n}_{2} .
$$

ПримеР 4. Рассмотрим смешанное состояние, которое описывается матрицей плотности

$$
\rho_{\mu}=\frac{1}{2}\left(\begin{array}{cccc}
0 & 0 & 0 & 0 \\
0 & 1 & -\mu & 0 \\
0 & -\mu & 1 & 0 \\
0 & 0 & 0 & 0
\end{array}\right)
$$


Для этого состояния матрица $P$ имеет вид

$$
P_{\mu}=\left(\begin{array}{ccc}
-\mu & 0 & 0 \\
0 & -\mu & 0 \\
0 & 0 & -1
\end{array}\right)
$$

В этом случае

$$
\begin{gathered}
\mathrm{RH}=2 \sqrt{1+\mu^{2}}, \quad \vec{n}_{1}=\frac{1}{\sqrt{2}}\left(\begin{array}{l}
0 \\
1 \\
1
\end{array}\right), \quad \vec{n}_{2}=\frac{1}{\sqrt{2}}\left(\begin{array}{c}
0 \\
-1 \\
1
\end{array}\right), \\
\vec{a}=\vec{n}_{1}, \quad \vec{b}=\left(\begin{array}{l}
0 \\
0 \\
1
\end{array}\right), \quad \vec{c}=\left(\begin{array}{l}
0 \\
1 \\
0
\end{array}\right), \quad \vec{d}=\vec{n}_{2} .
\end{gathered}
$$

ПримеР 5. Во всех предыдущих случаях мы рассматривали такие пары коррелированных спиновых состояний, в которых одночастичные состояния обладают определенной проекцией спина на одну и ту же ось. Рассмотрим состояние $\Psi_{0,0}^{\vec{k}_{1}, \vec{k}_{2}}$, аналогичное состоянию $\Psi_{0,0}$. Оно образовано двумя состояниями, одно из которых обладает определенным значением проекции спина на ось, задаваемую вектором $\vec{k}_{1}$, а второе - определенным значением проекции спина на ось, задаваемую вектором $\vec{k}_{2}$. В этом случае

$$
\vec{k}_{1}=\left(\begin{array}{l}
0 \\
1 \\
0
\end{array}\right), \quad \vec{k}_{2}=\left(\begin{array}{c}
0 \\
\sin \theta \\
\cos \theta
\end{array}\right) .
$$

Матрица плотности такого состояния имеет вид

$$
\rho_{\theta}=\frac{1}{4}\left(\begin{array}{cccc}
2 \sin ^{2} \frac{\theta}{2} & \sin \theta & -\sin \theta & 2 \sin ^{2} \frac{\theta}{2} \\
\sin \theta & 2 \cos ^{2} \frac{\theta}{2} & -2 \cos ^{2} \frac{\theta}{2} & \sin \theta \\
-\sin \theta & -2 \cos ^{2} \frac{\theta}{2} & 2 \cos ^{2} \frac{\theta}{2} & -\sin \theta \\
2 \sin ^{2} \frac{\theta}{2} & \sin \theta & -\sin \theta & 2 \sin ^{2} \frac{\theta}{2}
\end{array}\right),
$$

а матрица $P$ записывается как

$$
P_{\theta}=\left(\begin{array}{ccc}
-\cos \theta & 0 & -\sin \theta \\
0 & -1 & 0 \\
\sin \theta & 0 & -\cos \theta
\end{array}\right)
$$

Для этого состояния

$$
\begin{gathered}
\mathrm{RH}=2 \sqrt{2}, \quad \vec{n}_{1}=\left(\begin{array}{l}
0 \\
1 \\
0
\end{array}\right), \quad \vec{n}_{2}=\left(\begin{array}{c}
\cos \alpha \\
0 \\
\sin \alpha
\end{array}\right), \\
\vec{a}=\vec{n}_{1}, \quad \vec{b}=\frac{1}{\sqrt{2}}\left(\begin{array}{c}
\cos \alpha \\
1 \\
\sin \alpha
\end{array}\right), \quad \vec{c}=\frac{1}{\sqrt{2}}\left(\begin{array}{c}
-\cos \alpha \\
1 \\
-\sin \alpha
\end{array}\right), \quad \vec{d}=\vec{n}_{2},
\end{gathered}
$$

где $\alpha-$ произвольный угол. 
ПримеР 6. Рассмотрим процесс декогеренции, преобразующий состояние (48) в состояние, которое описывается матрицей плотности

$$
\rho_{\theta \mu}=\frac{1}{4}\left(\begin{array}{cccc}
2 \sin ^{2} \frac{\theta}{2} & \mu \sin \theta & -\mu \sin \theta & 2 \mu \sin ^{2} \frac{\theta}{2} \\
\mu \sin \theta & 2 \cos ^{2} \frac{\theta}{2} & -2 \mu \cos ^{2} \frac{\theta}{2} & \mu \sin \theta \\
-\mu \sin \theta & -2 \mu \cos ^{2} \frac{\theta}{2} & 2 \cos ^{2} \frac{\theta}{2} & -\mu \sin \theta \\
2 \mu \sin ^{2} \frac{\theta}{2} & \mu \sin \theta & -\mu \sin \theta & 2 \sin ^{2} \frac{\theta}{2}
\end{array}\right) .
$$

Для этого состояния матрица $P$ имеет вид

$$
P_{\theta \mu}=\left(\begin{array}{ccc}
-\mu \cos \theta & 0 & -\mu \sin \theta \\
0 & -\mu & 0 \\
\mu \sin \theta & 0 & -\cos \theta
\end{array}\right) .
$$

Если $\theta=\pi / 4$, можно показать, что

$$
\begin{gathered}
\mathrm{RH}=2 \sqrt{1+\mu^{2}}, \quad \vec{n}_{1}=\left(\begin{array}{l}
0 \\
1 \\
0
\end{array}\right), \quad \vec{n}_{2}=\left(\begin{array}{c}
\cos \alpha \\
0 \\
\sin \alpha
\end{array}\right), \quad \operatorname{tg} 2 \alpha=\frac{2 \mu}{1+\mu}, \quad \mu \neq 1, \\
\vec{a}=\vec{n}_{1}, \quad \vec{b}=\frac{1}{\sqrt{2}}\left(\begin{array}{c}
\cos \alpha \\
1 \\
\sin \alpha
\end{array}\right), \quad \vec{c}=\frac{1}{\sqrt{2}}\left(\begin{array}{c}
-\cos \alpha \\
1 \\
-\sin \alpha
\end{array}\right), \quad \vec{d}=\vec{n}_{2} .
\end{gathered}
$$

ПримеР 7. До сих пор мы рассматривали чистые максимально зацепленные состояния. Рассмотрим теперь состояние

$$
\Psi_{\varphi}=\cos \varphi|+\rangle|-\rangle+\sin \varphi|-\rangle|+\rangle,
$$

которое не является максимально зацепленным. Состоянию (52) соответствует матрица плотности

$$
\rho_{\varphi}=\left(\begin{array}{cccc}
0 & 0 & 0 & 0 \\
0 & \cos ^{2} \varphi & \cos \varphi \sin \varphi & 0 \\
0 & \cos \varphi \sin \varphi & \sin ^{2} \varphi & 0 \\
0 & 0 & 0 & 0
\end{array}\right)
$$

а матрица $P$ имеет вид

$$
P_{\varphi}=\left(\begin{array}{ccc}
\sin 2 \varphi & 0 & 0 \\
0 & \sin 2 \varphi & 0 \\
0 & 0 & -1
\end{array}\right) .
$$

Воспользовавшись формулой (30), получаем, что для состояния (52)

$$
\operatorname{RH}\left(\vec{n}_{1}, \vec{n}_{2}\right)=2 \sqrt{1+\sin ^{2} 2 \varphi} .
$$

При $\varphi=\pi / 4$ состояние (52) является максимально запутанным и соответствующая ему величина $\mathrm{RH}=2 \sqrt{2}$. Если же $\varphi=\pi / 2$, то оно становится факторизуемым и для него $\mathrm{RH}=2$. Мы видим, что величину $\mathrm{RH}$ можно использовать в качестве меры запутанности состояния. Подробно этот вопрос будет исследован в отдельной работе. 


\section{5. ЗАКЛЮЧЕНИЕ}

Итак, с помощью матрицы $P$, которая является редукцией матрицы плотности, можно сформулировать обобщенное неравенство БКХШХ, которое для каждого конкретного двучастичного спинового состояния при специальном выборе векторов $\vec{a}, \vec{b}, \vec{c}, \vec{d}$ превращается в точное равенство, и в этом смысле никогда не нарушается. Мы также показали, как с помощью этой матрицы $P$ можно находить такие векторы, и указали на связь величины RH с мерой запутанности двучастичного спинового состояния.

\section{Список литературы}

[1] J.S. Bell, Physics, 1 (1964), 195.

[2] J. F. Clauser, M. A. Horne, A. Shimony, R. A. Holt, Phys. Rev. Lett., 23 (1969), 880.

[3] S. J. Freedman, J. F. Clauser, Phys. Rev. Lett., 28 (1972), 938.

[4] A. Aspect, P. Grangier, G. Roger, Phys. Rev. Lett., 47 (1981), 460.

[5] A. Aspect, P. Grangier, G. Roger, Phys. Rev. Lett., 49 (1982), 91.

[6] A. Aspect, J. Dalibard, G. Roger, Phys. Rev. Lett., 49 (1982), 1804.

[7] Z. Y. Ou, L. Mandel, Phys. Rev. Lett., 61 (1988), 50.

[8] T. E. Kiess, Y.H. Shih, A. V. Sergienko, C. O. Alley, Phys. Rev. Lett., 71 (1993), 3893.

[9] В. А. Андреев, В. И. Манько, ТМФ, 140 (2004), 284.

[10] I. V. Volovich, Bell's theorem and locality in space, quant-ph/0012010.

[11] L. Accardi, M. Regoli, Locality and Bell's inequality, quant-ph/0007005.

[12] М.Б. Менский, Квантовые измерения и декогерениия, Физматлит, М., 2001.

[13] А.С. Холево, Введение в квантовую теорию информации, Соврем. матем. физика. Проблемы и методы, 5, МЦНМО, М., 2002.

[14] A. Khrennikov, Found. Phys., 32 (2002), 1159.

[15] A. Khrennikov, I. Volovich, Local realism, contextualism and loopholes in Bell's experiments, quant-ph/0212127.

[16] А. Ю. Хренников, Неколмогоровские теории вероятностей и квантовая физика, Физматлит, М., 2003.

[17] A. Khrennikov, I. V. Volovich, "Quantum nonlocality, EPR model, and Bell's theorem", Proc. III Int. Sakharov Conf. on Physics, vol. 2 (Moscow, Russia, 2002), eds. A. Semikhatov, M. Vasiliev, V. Zaikin, World Sci., Singapore, 2003, 260.

[18] V. A. Andreev, V.I. Man'ko, "The quantum tomography representation of Bell-CHSH inequalities", Quantum Theory: Reconsideration of Foundations - 2 (Växjö, Sweden, 2003), Math. Model. Phys. Eng. Cogn. Sci., 10, ed. A. Khrennikov, Växjö Univ. Press, Växjö, 2004, 47 .

[19] В. А. Андреев, В. И. Манько, О. В. Манько, Е. В. Щукин, ТМФ, 146 (2006), 172.

[20] В. А. Андреев, В. И. Манько, Писъма в ЖЭТФ, 72 (2000), 130.

[21] V.A. Andreev, V. I. Man'ko, Phys. Lett. A, 281 (2001), 278.

[22] R. F. Werner, Phys. Rev. A, 40 (1989), 4277. 